\title{
Principles of a gas filled magnetic spectrometer for fission studies
}

\author{
H. Faust ${ }^{1, \mathrm{a}}$, A. Chebboubi², G. Kessedjian², C. Sage ${ }^{2}$, U. Köster ${ }^{1}$, and A. Blanc ${ }^{1}$ \\ ${ }^{1}$ Institut Laue-Langevin, 6 rue Jules Horowitz, 38042 Grenoble, France \\ ${ }^{2}$ LPSC Grenoble, CNRS/IN2P3, 53 rue des Martyrs, 38026 Grenoble, France
}

\begin{abstract}
The spectroscopy of the prompt gamma decay from fission products gives information on the entry states, e.g. distribution functions for excitation energy and spin, and therefore a direct link to the fission process itself. This type of spectroscopy is, however, only possible when a filter can be constructed which allows setting a gate to the gamma-spectrum in a narrow region in mass and nuclear charge, as well as on the total excitation energy of the fragment split under investigation. A possible configuration of a prompt gamma-ray spectrometer consist of a gamma-ray array composed of high resolution germanium detectors, coupled to a gas filled magnet. We will outline the principles for a gas filled magnetic spectrometer for fission product spectroscopy. In particular the focusing characteristics of such a device, which are valid for particles in the velocity regime of $\mathrm{E} / \mathrm{A}<1 \mathrm{MeV} / \mathrm{amu}$, will be addressed. First experiments on the LOHENGRIN spectrometer in Grenoble investigating on the behavior of fission products in gas filled magnets have been performed, and have validated the experimental approach to the nuclear fission process with such a device.
\end{abstract}

\section{Introduction}

Much work has been done over the last 75 years to measure observables in nuclear fission, mostly fission product mass, nuclear charge, and kinetic energy. The distributions of these observables are strongly dependent on mass and charge of the fissioning compound system, and the excitation energy which is given to the system to induce the fission process. Accessible are mostly secondary fission product distributions, after the emission of prompt neutrons and gamma rays. Standard measurements of mass and kinetic energy are done by measuring the kinetic energy of both fragments with the help of ionisation chambers or surface barrier detectors. By the measurement of the ratio of the two kinetic energies, and the assumption that both fragment masses add up to the compound nucleus mass, a rough idea of the mass distribution can be obtained, as well as a rough idea about the total kinetic energies by doing assumptions on the neutron evaporation process. A step further to obtain distribution functions for fission products is the combined measurement of the two velocities and the two kinetic energies of both fission products, which allows the reconstruction of the primary quantities of mass and total

\footnotetext{
ae-mail: faust@ill.fr
}

This is an Open Access article distributed under the terms of the Creative Commons Attribution License 2.0, which permits unrestricted use, distribution, and reproduction in any medium, provided the original work is properly cited. 
kinetic energy. In general all these measurements suffer from limited resolution functions for the kinetic energy and the time of flight, and much work is devoted to improve the numbers with the aim to get mass resolutions allowing obtaining fission yield mass by mass. A further aim of these measurements is the determination of the fission product nuclear charge, which is in principle accessible by recording the ionisation density of the fission particle track in the ionisation chamber. However, present day techniques give only access to the determination of nuclear charges up to $\mathrm{Z}=40$ with this method.

A powerful way to the determination of distribution functions for the secondary fission product is the use of sophisticated mass spectrometers, like the LOHENGRIN facility in Grenoble /France. Here full distribution functions for mass and nuclear charge, as well as kinetic energy can be measured due to mass resolutions which reach $\mathrm{A} / \Delta \mathrm{A}=600$, nuclear charge resolutions of order $\mathrm{Z} / \Delta \mathrm{Z}=34$, and kinetic energy resolutions of $\mathrm{E} / \Delta \mathrm{E}=1000$. These values allowed in the past to determine distribution functions for mass, charge and energy for most actinides with fission cross sections for thermal neutrons in the 1 barn region. Unfortunately, due to the limited resolution, charge distributions can only be measured for the light fission products. For the heavy fission product region gamma spectroscopy is applied, and the yield Y(A, Z, Ekin) is obtained by the detection of characteristic gamma rays following beta decay, see [1]. This method is however limited due to un-complete knowledge of branching ratios of the process, and by sometimes too long beta decay times. Nevertheless, about $85 \%$ off the fission yield in the heavy fission product wing can be determined via this technique. Last but not least the advent of inverse reactions constitutes a powerful tool to obtain distribution functions for fission reactions mostly from neutron deficient compound nuclei.

The above techniques allow for the determination of mass, charge and kinetic energy of the fission products, as well as to the distribution function for the total excitation energy TXE, which is determined by a measurement of the total kinetic energy TKE, and by the knowledge of the Q-value of the reaction. This supposes that the primary mass and charge split its known.

We advocate here a different approach for measuring fission observables. Due to the availability of large germanium arrays it appears nowadays possible to determine fission yield by the detection of characteristic prompt gamma rays, if it is assumed that the statistical model applies to nuclear fission. Then fission follows the rules of the well known statistical model, and the entry states for fission fragments in term of excitation energy and spin can be calculated by knowing the nuclear temperature, which can be determined via a measurement of the fragment kinetic energy. From the entry states the distribution functions for prompt neutron and gamma emission for the individual fragment are then found by further applying the statistical model.

The applicability of the statistical model for the nuclear fission process can be verified by comparing the known yield distribution of a standard reaction like ${ }^{235} U(n, f)$ with the yield determination from prompt gamma rays. We will in the following elaborate on the theoretical basis for such an approach, and present a spectrometer which allows the measurement of $Y\left(A, Z, E_{k i n}, k T\right)$ for thermal neutron induced fission, but also for fission reactions following fast neuron capture or charged particle reactions.

\section{Excitation and decay of fission products}

Following the fission process the fragments are excited to an excitation energy $\mathrm{E}^{*}$ and a spin J. If the thermodynamic model is applied the distribution function for excitation energy and spin for one fission species $(\mathrm{A}, \mathrm{Z})$ is given by the level density at $\mathrm{E}^{*}$ and by the Boltzmann factor, which contains the temperature of the microcanonical ensemble. The ensemble includes all fission products of the same $(\mathrm{A}, \mathrm{Z})$, and stemming from the same reaction (here thermal neutron induced fission). The distribution function reads, with $\rho\left(\mathrm{E}^{*}, \mathrm{~J}\right)$ being the level density at excitation $\mathrm{E}^{*}$ for spin $\mathrm{J}$

$$
P\left(E^{*}, J\right) d E^{*}=\frac{1}{N} * \rho\left(E^{*}, J\right)^{*} e^{-E^{*} / k T} d E^{*} .
$$


The above function can be further separated into two functions, the function $\Theta\left(\mathrm{E}^{*}\right)$ which contains the excitation distribution of the fragments, and further $\Phi(\mathrm{J})$ which contains the spin:

$$
P\left(E^{*}, J\right)=\Theta\left(E^{*}\right) \cdot \Phi(J) .
$$

Bethe [2] has shown that, based on the sequence of shell model states, the spin distribution can be expressed by the following equation

$$
\Phi_{J}=\frac{2 J+1}{2 \sigma^{2}} \exp \left(-\frac{J(J+1)}{2 \sigma^{2}}\right) .
$$

Here $\sigma$ is the spin cut off parameter which contains the relevant physical quantities, e.g. the level density parameter a, the temperature of the nuclear ensemble kT, and the mass of the fragment A (see [3]).

$$
\sigma^{2}=0.0888 \cdot a_{1,2} \cdot k T \cdot A_{1,2}^{2 / 3} \text {. }
$$

If the nuclear temperature $\mathrm{kT}$ is known, the entry states for fission products in terms of excitation and spin can be directly evaluated. The temperature parameter which is needed in the expression for excitation energy and spin can be extracted from the measurement of the total excitation energy TXE, which, by applying momentum and energy conservation laws, can in turn be determined from the total kinetic energy TKE, or alternatively by a measurement of the single fragment kinetic energy $E_{k i n}$ :

$$
\begin{array}{r}
T X E=Q-T K E \\
T K E=E_{k i n 1}+E_{k i n 2} .
\end{array}
$$

In the thermodynamic model the inverse of the temperature of the system is calculated from the derivative of the entropy with respect to the total kinetic energy. The entropy of a nuclear system is given by the natural logarithm of the level density, $S=\ln (\rho)$. It is in general assumed that entropy is additive, $S_{\text {tot }}=S_{1}+S_{2}$, which leads to the following expression for the available states in a system which is composed of two fission fragments:

$$
\rho_{\text {tot }}(T X E)=\int_{E_{1}^{*}=0}^{T X E} \rho\left(E_{1}^{*}\right) \cdot \rho\left(T X E-E_{1}^{*}\right) d E_{1}^{*} .
$$

The above expression counts all the possible combinations which can be reached for a given TXE. Knowing the temperature, the entry state distributions for the fission fragment is evaluated. By further applying the statistical model prompt neutron and gamma decay can be calculated.

On the general accepted assumption that the entry state distribution and the decay path of an excited fragment is only a function of the temperature, the yield from an unknown system can be determined via the spectroscopy of prompt gamma rays if a calibration from a standard system is available, e.g. for ${ }^{235} U$.

\section{Basic consideration for gas filled magnet devices}

Prompt gamma decay gives an access to excitation energy and spin distributions in fragments, and by means of calibration from specific gamma intensities the yield of a fragment as function of (A,Z,Ekin,kT) can be extracted. Measuring the individual gamma decay of individual fission fragments is, however, not a simple task. The fission reaction produces many hundreds of different fission products, and the assignment of specific gamma quanta to a fragment with (A,Z) is only possible by multiple coincidence techniques, or, alternatively, by providing a filter which allows gating the prompt gamma spectrum for a narrow region of nuclear mass and charge. The aim is here to design a spectrometer to be used as a filter for a restricted region of fission products. It will be shown that a gas filled magnet provides the right characteristics for such a filter. 


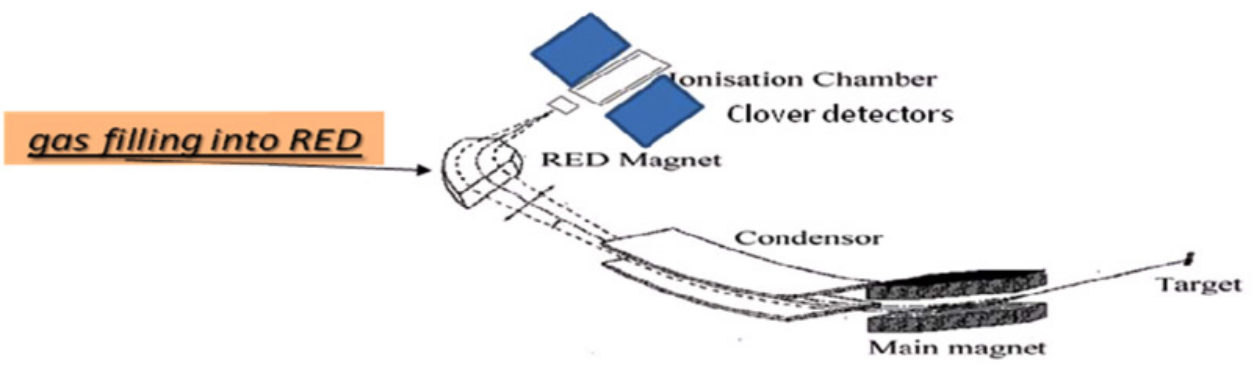

Figure 1. Experimental arrangement for the measurement of the focalizing properties of gas filled magnets.

The bending radius of a charged particle in a gas filled magnet is given by the Lorentz force

$$
B \rho \sim \frac{\langle v\rangle}{\langle q\rangle} A .
$$

Here $\rho$ is the bending radius at the field $\mathrm{B}, \mathrm{A}$ is the particle mass, and $\langle\mathrm{v}\rangle$ and $\langle\mathrm{q}\rangle$ are the mean values of particle velocity and particle ionic charge at a given position in the magnet gap. The gas filling in the magnet gap leads to collisions of the particle with the gas atoms. The energy loss due to the collisions is according to Bethe-Bloch in the non-relativistic case, which is a good approximation for fission products

$$
\frac{d E}{d x} \sim \frac{\langle q\rangle^{2}}{\langle v\rangle^{2}} \ln \left(v^{2}\right) .
$$

Along the particle track through the magnet bending radius and stopping will be determined by the ratio of the mean values of velocity over ion-charge. Already Bohr has argued that in the energy regime

$$
1 \leq \frac{v}{v_{0}} \leq Z^{2 / 3}
$$

velocity and mean value of the ionic charge are strongly correlated, and follow the expression

$$
\frac{\langle v\rangle}{\langle q\rangle} \propto Z^{-1 / 3} .
$$

Any change in the ion velocity in the gas due to stopping leads to a change in q, so that their ratio stays constant. Inserted in bending radius and stopping this leads to

$$
\begin{gathered}
B \rho \propto A \cdot Z^{-1 / 3} \\
\frac{d E}{d x} \propto Z^{2 / 3} \cdot \ln v^{2} .
\end{gathered}
$$

The above equations show that the particle track in a gas filled magnet, given by the $B \rho$ value, is independent of the particle kinetic energy, with the consequence that a gas filled device will focus all fission products of the same value $(\mathrm{A}, \mathrm{Z})$ to the same position.

We have performed experiments on the LOHENGRIN spectrometer in Grenoble to investigate to which level the Bohr estimate is valid, and what the resolutions are which can be reached for such a type of spectrometer. For this purpose the last magnetic field on the spectrometer was filled with a stopping gas, and we used clover detectors to identify fission products selected in nuclear mass and nuclear charge. In order to identify clearly the isotope delivered by the LOHENGRIN-spectrometer we have chosen nuclei with microsecond isomeric states, e.g. ${ }^{88} \mathrm{~B},{ }^{98} \mathrm{Y},{ }^{132} \mathrm{Te},{ }^{136} \mathrm{Xe}$. Due to the short lifetime of the isomeric states a gate can be set on the arrival of the ion in the ionisation chamber, and the isomeric decay detected nearly background-free. We used different gas fillings, from $\mathrm{He}$ to 


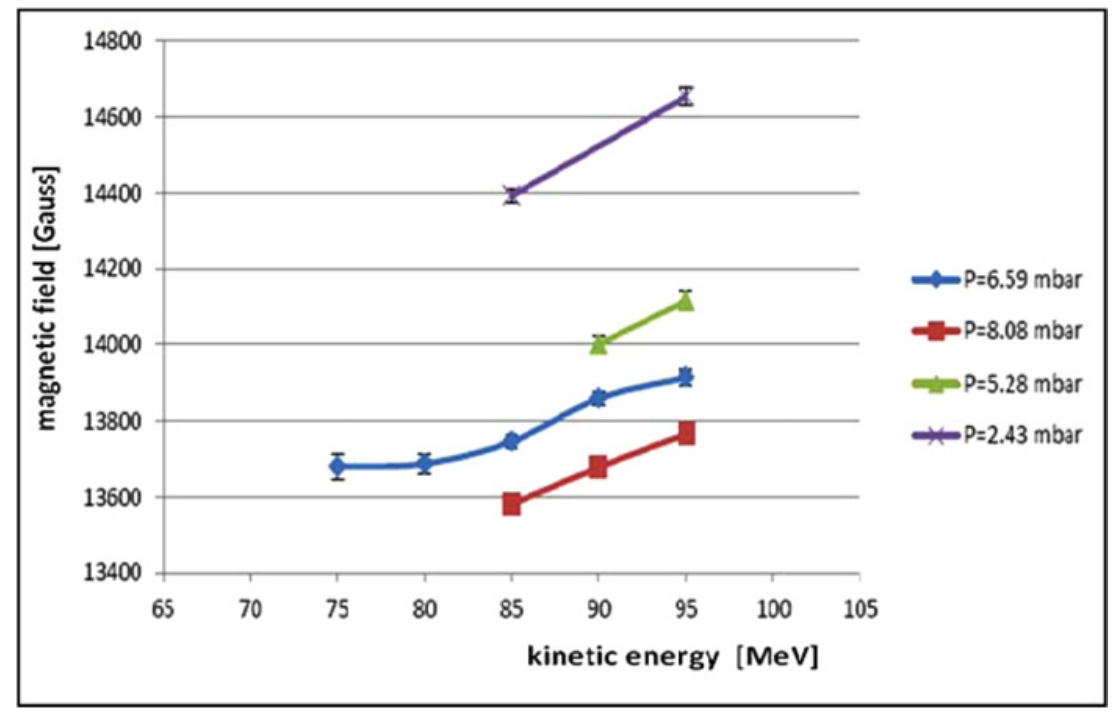

Figure 2. Mean values of the magnetic field required to focalize ${ }^{98} Y$ ions in $N_{2}$ at different gas pressures and different incoming particle energies.

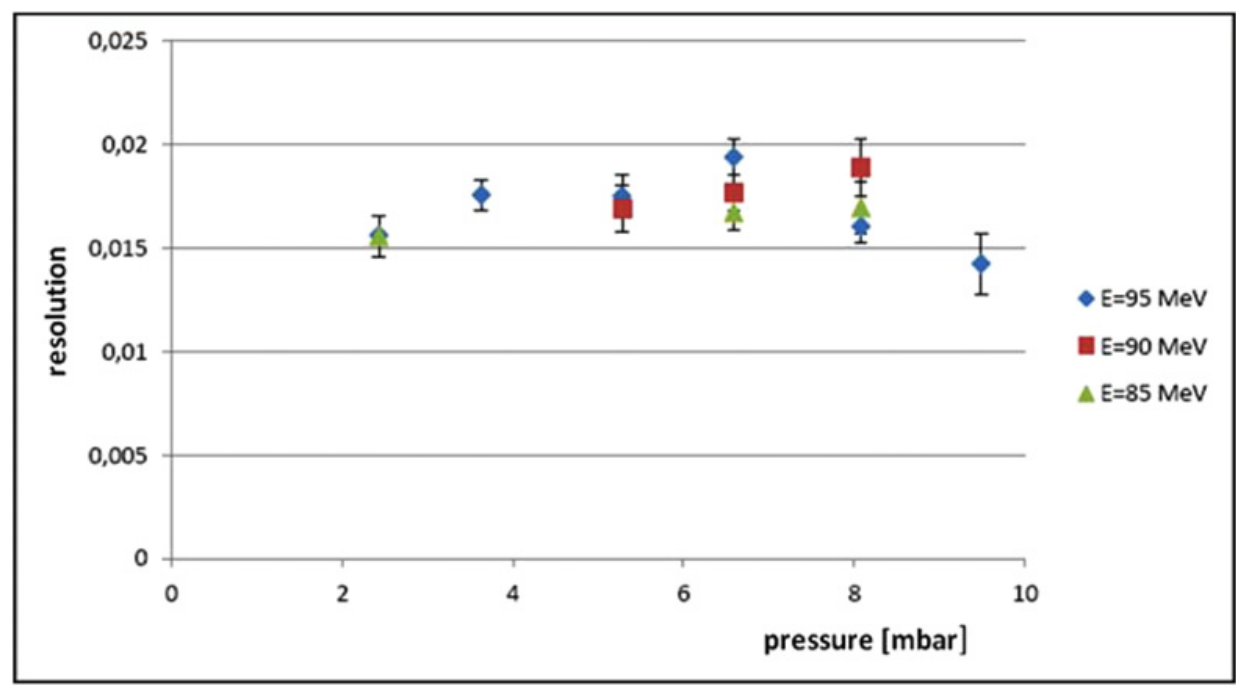

Figure 3. Values for the resolution $\Delta B \rho / B \rho$ for ${ }^{98} Y$, measured at different gas pressures in $N_{2}$, and at different incoming fragment kinetic energies.

$N_{2}, A r$, and isobutane, at different pressures to cover the whole range of experimental possibilities. The experimental set-up is shown in Figure 1. In Figure 2 we show one of the results obtained. Here the mean value of the magnetic field needed to bend the ions into the ionisation chamber is recorded for ${ }^{98} Y$, at different gas pressures for $N_{2}$. The plot demonstrates that the required magnetic field strength, which is proportional to the $B \rho$ value, is nearly constant over a wide range of the incoming particle velocity. The mean values for $B \rho$ change only by about $1.4 \%$ when the incoming energy of the ion is changed by 
about $20 \%$. Furthermore there is a pressure dependence on the mean value of $B \rho$, which was expected from the assumption that at higher gas pressures the mean ionic charge state increases slightly.

Figure 3 shows the resolution $\Delta B \rho / B \rho$ reached in our gas filled device. It demonstrates that the resolution is largely independent of the gas pressure and the velocity of the incoming ion. Our experiments with different gases have shown that the resolution of about 1.5 to $2 \%$ seems to be a natural limit for gas filled devices, and seems to be inherent in the charge changing processes along the trajectory of the ion in the gas.

\section{Conclusions}

According to our experiments with gas filled magnetic devices the following statements can be made:

- the Bohr criterion appears to be fully applicable to fragments in the fission product regime. Here the focussing properties of a gas filled magnet are independent of the particle velocity. They are also independent of the nature of the gas filling (for gases heavier than Helium)

- the $B \rho$ resolution which can be reached with a gas filled device is about $1.5-2.0 \%$, independent of the gas filling and over a large pressure range. This seems to be a general limit for gas filled devices. It seems therefore not useful to design gas filled spectrometers which aim at a better resolution

- due to this limiting value for resolution in $B \rho$ the magnet design can be optimized for a large solid angle

- Liouvilles theorem is not longer valid for gas filled devices, because the phase space is not constant due to stopping. However, the design-rules for magnetic spectrometers without gas are valid also for gas filled systems (focusing characteristics, double focusing by fringing fields)

- due to the large solid angle which can be reached it seems advantageous to design a high efficiency magnetic device (eventually by using a magnet design with a 1/R-field).

\section{References}

[1] A.Bail et al, Phys. Rev.C14, 034605 (2011)

[2] H.A. Bethe, Rev. Mod. Phys. 9 (1937) 69

[3] A. Gilbert and A.G.W. Cameron, Canadian Journal of Physics 43 (1965) 446 